\title{
Reflected scatterometry for noninvasive interrogation of bacterial colonies
}

\author{
Huisung Kim \\ Iyll-Joon Doh \\ Jennifer Sturgis \\ Arun K. Bhunia \\ J. Paul Robinson \\ Euiwon Bae
}




\title{
Reflected scatterometry for noninvasive interrogation of bacterial colonies
}

\author{
Huisung Kim, ${ }^{a, \dagger}$ lyll-Joon Doh, ${ }^{a, \dagger}$ Jennifer Sturgis, ${ }^{b}$ Arun K. Bhunia, ${ }^{c}$ J. Paul Robinson,,d and Euiwon Bae ${ }^{a, \star}$ \\ aPurdue University, School of Mechanical Engineering, Applied Optics Laboratory, West Lafayette, Indiana 47907, United States \\ ${ }^{b}$ Purdue University, Department of Basic Medical Sciences, West Lafayette, Indiana 47907, United States \\ 'Purdue University, Molecular Food Microbiology Laboratory, Department of Food Science, West Lafayette, Indiana 47907, United States

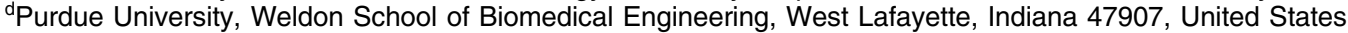

\begin{abstract}
A phenotyping of bacterial colonies on agar plates using forward-scattering diffraction-pattern analysis provided promising classification of several different bacteria such as Salmonella, Vibrio, Listeria, and E. coli. Since the technique is based on forward-scattering phenomena, light transmittance of both the colony and the medium is critical to ensure quality data. However, numerous microorganisms and their growth media allow only limited light penetration and render the forward-scattering measurement a challenging task. For example, yeast, Lactobacillus, mold, and several soil bacteria form colorful and dense colonies that obstruct most of the incoming light passing through them. Moreover, blood agar, which is widely utilized in the clinical field, completely blocks the incident coherent light source used in forward scatterometry. We present a newly designed reflection scatterometer and validation of the resolving power of the instrument. The reflectance-type instrument can acquire backward elastic scatter patterns for both highly opaque media and colonies and has been tested with three different bacterial genera grown on blood agar plates. Cross-validation results show a classification rate above 90\% for four genera. ๑ 2016 Society of Photo-Optical Instrumentation Engineers (SPIE) [DOI: 10.1117/1.JBO.21.10.107004]
\end{abstract}

Keywords: bacteria identification; diffraction; light scattering; backward-scattering; instrumentation and measurement. Paper 160220RRR received Apr. 8, 2016; accepted for publication Oct. 6, 2016; published online Oct. 24, 2016.

\section{Introduction}

A laser-based light-scatter technique was reported for label-free phenotypic screening of bacterial colonies ${ }^{1}$ and has shown promising classification results for several bacteria, including Salmonella, ${ }^{2}$ Vibrio, ${ }^{3}$ Listeria,${ }^{4}$ Campylobacter,${ }^{5}$ and Escherichia coli. ${ }^{6}$ As the need for rapid identification and classification of microbial organisms increases in various fields such as food security, clinical studies, and biosurveillance, label-free optical diagnostics has been studied by several research groups owing to several merits of this technology. ${ }^{7-9}$ To provide more robust and accurate screening of these patterns, machine-learning techniques such as support vector machines (SVMs) were applied to features extracted from scatter patterns. ${ }^{10}$ In addition, the optical origin of the patterns was investigated based on elastic-lightscatter phenomena for a single wavelength, ${ }^{11}$ multiwavelengths, ${ }^{12}$ and a speckle analysis. ${ }^{13}$

One drawback of the current system is that the scattered signal must transmit through the colony and growth medium. Therefore, the current ELS system has shown limited performance success for bacterial colonies with high opacity or highly reflective growth media, such as yeast colonies or blood agar medium, respectively. In particular, blood agar medium is widely used in clinical and veterinary medicine to isolate specific bacteria that generate hemolysis. ${ }^{14,15}$ Media or colonies with high opaqueness block the incoming light and do not allow photons to penetrate; as a consequence, the forward-scatter patterns cannot be observed by the sensor. Therefore, to expand the applicability of the light-scattering technology into clinical and

\footnotetext{
*Address all correspondence to: Euiwon Bae, E-mail: ebae@purdue.edu
}

†These authors contributed equally. veterinary medicine, we have developed a reflection-type of scatterometer that can generate patterns in such circumstances. This technique involves reflected elastic scattering, which is designed to capture the patterns above the colonies. The reflectance-based method utilizes a green laser diode (532 nm), an R50:T50 beam-splitter, a large nonreflective screen placed directly above the colonies, and a high-resolution camera to capture patterns on the screen. Owing to the larger scattering angle in the backward direction, the new system was designed to effectively capture the larger scatter patterns. A prototype system was tested with four different bacterial genera $(E$. coli K12, Listeria innocua F4244, Salmonella typhimurium, and Staphylococcus aureus) grown on a blood agar plate with 5\% sheep blood. Despite the opacity of the medium, the unique patterns of each genus can be observed, analyzed, and classified.

\section{Materials and Methods}

\subsection{Design of Reflective Scatterometer}

Figure 1(a) shows a schematic diagram of a newly designed reflective BARDOT system. A high-resolution digital camera (D810, Nikon Corp., Tokyo, Japan) with $7360(\mathrm{H}) \times$ 4912(V) pixels and an AF-S NIKKOR $85 \mathrm{~mm} \mathrm{f} / 1.8 \mathrm{G}$ lens (Nikon Corp., Tokyo, Japan) is positioned over the Petri dish at a distance of $490 \mathrm{~mm}\left(19.5^{\prime \prime}\right)$, measured from the top surface of the Petri dish to the surface of the camera image plane [Fig. 1(b)]. Exposure program, ISO, F-number, exposure bias value, and focal length of the camera are fixed in manual mode, 800 , f $8,0.00 \mathrm{eV}$, and $85 \mathrm{~mm}$, respectively. A black

$1083-3668 / 2016 / \$ 25.00$ @ 2016 SPIE 


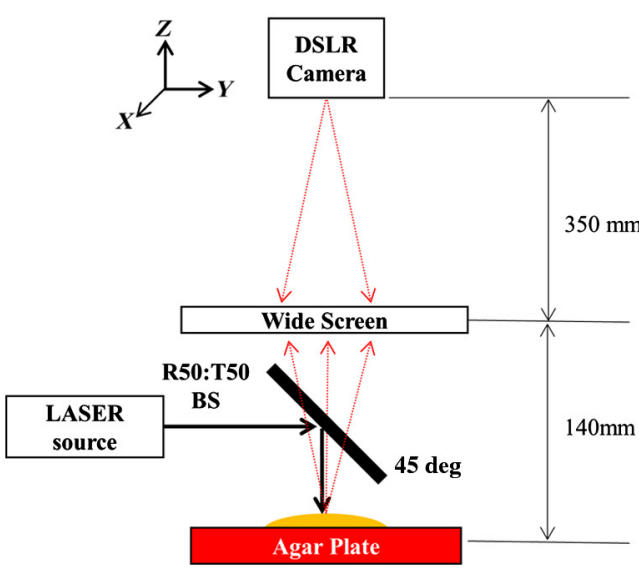

(a)

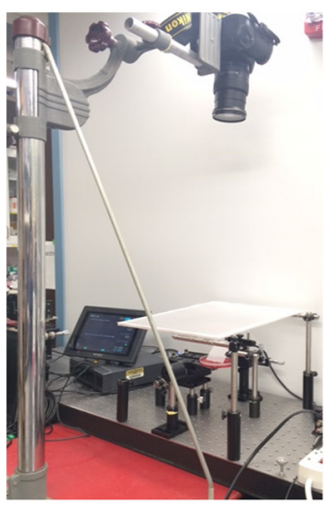

(b)

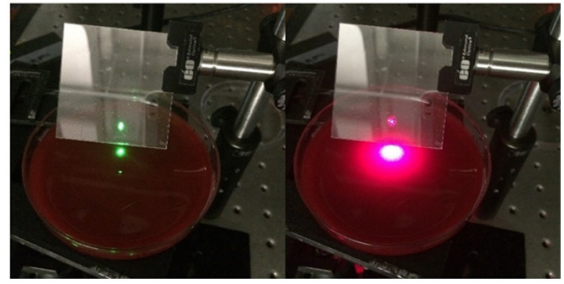

(c)

Fig. 1 (a) Schematic diagram and experiment setup. Due to the oversized diameter of the backwardscatter patterns, wide screen, and 50:50 (R/T) BS was used to realize the reflection setup. (b) Photograph of the actual setup. (c) Comparison of the different wavelength lasers on the blood agar media: (left) $532 \mathrm{~nm}$ and (right) $635 \mathrm{~nm}$. As clearly seen, 635-nm wavelength light diffusively penetrates the blood agar and spreads out inside the media rather than reflecting off the surface.

curtain covers the instrument completely to block unexpected environment light, and a 2 -s shutter speed is used for the experiments. The shutter time is selected based on scattering pattern intensity, tested over a variety of shutter time options from $0.3 \mathrm{~s}$ to $3 \mathrm{~s}$. A collimated, circular $5-\mathrm{mW}$ beam from a $532-\mathrm{nm}$ laser diode module (GLM-R1IB-05 Innovam Lasers, Montreal, Canada) acts as the light source [Fig. 1(c)]. As shown in Fig. 1(a), an R50:T50 plate beam splitter (BS) (BTF-VIS-30SQW-5001M-C IDEX Optics \& Photonics Marketplace, Albuquerque, New Mexico) is located over the agar plate at a 45-deg angle to deflect the incoming light to the sample. To avoid interference from an edge of the BS for the pattern, a $50 \times 50 \times 1 \mathrm{~mm}$ rectangular BS is selected. The light from the source is reflected and backscattered by the bacterial colony and forms a pattern on a screen located in between the BS and the camera; this pattern is captured by the camera as an RAW format for analysis.

As with the forward scatterometer, direct scatter-pattern capture without passing through any optical component guarantees the best quality; however, the screen is adapted owing to the larger pattern size produced by a larger diffraction angle (scattering angle) and the spatial limitation of the commercial large-area CMOS sensors. The screen was a diffuser film $279.4 \times 355.6 \mathrm{~mm}\left(11^{\prime \prime} \times 14^{\prime \prime}\right)$ mounted in a monitor. Since the quality of the screen-captured pattern from the reflectiontype instrument is also suitable for the custom-built patternanalyzer program, we utilized the screen rather than installing an expensive wide-imaging sensor. The screen is located $140 \mathrm{~mm}\left(\sim 5.5^{\prime \prime}\right)$ above the sample to ensure the high spatial resolution and quality of the scatter patterns.

\subsection{Sample Preparation}

E. coli $\mathrm{K} 12$, L. innocua, S. typhimurium, and S. aureus were selected as model organisms. All genera were prepared by subculturing from frozen stocks stored in a freezer that was kept at $-80^{\circ} \mathrm{C}$. From the frozen stock, each genus was cultured on trypticase soy agar (Bacto ${ }^{\mathrm{TM}}$, BD Diagnostics, Sparks, Maryland), and incubated at $37^{\circ} \mathrm{C}$ until colonies were able to be visibly located. One of the colonies was randomly selected to be subcultured on blood agar. The selected culture was then diluted serially two to three times with $100 \mu \mathrm{L}$ for each dilution. From the final diluted tube, $50 \mu \mathrm{L}$ was surface plated on a trypticase soy agar plate with $5 \%$ sheep blood $(100 \mathrm{~mm} \times 15 \mathrm{~mm})$, (TSA IITM, BD Diagnostics, Sparks, Maryland) to obtain bacterial colony counts of 50 to $100 \mathrm{CFU} /$ plate. The plates were incubated at $37^{\circ} \mathrm{C}$ until the colonies reached a diameter of 700 to $900 \mu \mathrm{m}$. Colony diameter and elevation were measured using both a confocal microscope equipped with a Leica DFC310 FX CCD camera and Leica Application Suite V4.20 build 607 (all from Leica Microsystems, Bannockburn, Illinois) using a $2 \times$ objective, and a custom-built colony morphology analyzer. $^{16}$

\subsection{Optical Modeling}

To predict the pattern size, a geometric optics was utilized as a first-order approach. It is assumed that only a reflection by the curvature of the colony is deflecting the incoming light. ${ }^{17}$ Figure 2 shows the coordinate system of the analysis. Subscripts s, a, and i denote a source, an aperture, and an image plane, respectively. 


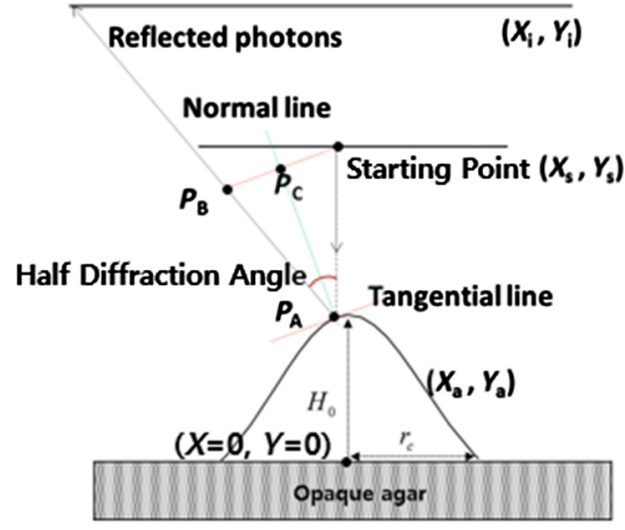

Fig. 2 Schematic diagram of the coordinate definition. Incoming photons $\left(X_{\mathrm{s}}, Y_{\mathrm{s}}\right)$ from source are reflected from the colony surface $\left(X_{\mathrm{a}}, Y_{\mathrm{a}}\right)$ where its profile is defined as Eq. (1). At each incidence of reflected photons, the tangential and respective normal line is computed by $\mathrm{P}_{\mathrm{A}}$ and $\mathrm{P}_{\mathrm{C}}$. Then intersection between $\mathrm{P}_{\mathrm{B}}$ and imaging plane $\left(X_{\mathrm{i}}, Y_{\mathrm{i}}\right)$ was calculated to estimate the maximum half diffraction angle.

A bacterial colony is considered to be an optical reflector like a convex mirror. The curvature of the colony is modeled as a Gaussian profile with tailing edge as

Colony profile $\left(x_{\mathrm{a}}, y_{\mathrm{a}}\right)=H_{0} \exp \left[-\frac{\left(x_{\mathrm{a}}^{2}+y_{\mathrm{a}}^{2}\right)}{r_{\mathrm{c}}^{2}}\right]$,

where $H_{0}$ is defined as the center height of the colony. For the tailing-edge representation, an effective radius of the colony, $r_{\mathrm{c}}$, is defined as

$r_{\mathrm{c}}=\frac{D}{2 F}$,

where $D$ is the measured diameter of the colony, and $F$ is a factor (1.6) in the divisor to render a $1 / e^{3}$ radius. The incoming light is assumed to be collimated light that covers the whole area of the colony. When one of the incoming photons reaches the point $P_{\mathrm{A}}$ located on the curvature of the colony, it will be reflected back to the image plane. The normal line in Fig. 2 is derived as

$y=N_{\mathrm{s}} x+y_{\mathrm{a}}-x_{\mathrm{a}} N_{\mathrm{s}}$,

where $N_{\mathrm{s}}$ is defined as the slope of the normal line. A point $P_{\mathrm{C}}$ is the bisector between the starting point and $P_{\mathrm{B}}$ is on the reflected photon trajectory. The point $P_{\mathrm{C}}$, which lies on the normal line, can be expressed in terms of $x$ and $y$ coordinates of $P_{\mathrm{B}}$ and starting point as shown in Eq. (4). The slope between the starting point and $P_{\mathrm{B}}$ is perpendicular to that of the normal line. This can be expressed as

$P_{\mathrm{c}}=\left(\frac{x_{\mathrm{s}}+x_{\mathrm{b}}}{2}, \frac{y_{\mathrm{s}}+y_{\mathrm{b}}}{2}\right)$,

$\frac{y_{\mathrm{b}}-y_{\mathrm{s}}}{x_{\mathrm{b}}-x_{\mathrm{s}}}=-1 / N_{\mathrm{s}}$.

After substituting Eq. (4) in Eq. (3) and organizing Eq. (5), the derived equation as a matrix form with respect to $\left(x_{\mathrm{b}}, y_{\mathrm{b}}\right)$ is obtained. $\left[\begin{array}{cc}1 & N_{\mathrm{s}} \\ N_{\mathrm{s}} & -1\end{array}\right]\left[\begin{array}{l}x_{\mathrm{b}} \\ y_{\mathrm{b}}\end{array}\right]=\left[\begin{array}{c}N_{\mathrm{s}} y_{\mathrm{s}}+x_{\mathrm{s}} \\ -N_{\mathrm{s}} x_{\mathrm{s}}+y_{\mathrm{s}}-2 y_{\mathrm{a}}+2 N_{\mathrm{s}} x_{\mathrm{a}}\end{array}\right]$,

$\left(x_{\mathrm{b}}, y_{\mathrm{b}}\right)$, coordinate point of $P_{\mathrm{B}}$ that is on the reflected photon trajectory, is computed from Eq. (6). Since $P_{\mathrm{A}}$ and $P_{\mathrm{B}}$ are on the reflected line, the line equation for the reflected line is derived as

$x_{\mathrm{i}}=\frac{x_{\mathrm{b}}-x_{\mathrm{a}}}{y_{\mathrm{b}}-y_{\mathrm{a}}}\left(y_{\mathrm{i}}-y_{\mathrm{b}}\right)+x_{\mathrm{b}}$.

Since the image plane where the reflected pattern is captured is located at $y_{\mathrm{i}}$, the half-scatter angle $x_{\mathrm{i}}$ is computed by Eq. (7). The light source is assumed to be a round, collimated $2 \mathrm{~mm}$ laser beam, which illuminates the whole colony perpendicularly and does not block any light reflected from the colony. The distance from the colony to the image plane where the screen is located is set as $127 \mathrm{~mm}$. The angle between the incident light (perpendicular to the ground) and reflected light at the most outer bound is considered the maximum half diffraction angle, which determines the pattern size. The aspect ratio (a colony elevation and diameter ratio), which plays a major role to determine the slope of the colony curvature, is fixed as $1: 10$ for the simulation of Figs. 5(a) and 5(b). ${ }^{11,18}$ The elevation and diameter of the colony are set as 100 and $1000 \mu \mathrm{m}$, respectively. In studying the relationship between aspect ratio and diffraction angle, the aspect ratio varied from 0.015 to 1.5 .

\subsection{Time-Lapse Backward-Scatter Patterns}

All four bacteria samples were incubated (I2400 Incubator Shaker, New Brunswick Scientific, Edison, New Jersey) and $\sim 15$ colonies per plate were measured. The first measurement was taken when E. coli, S. typhimurium, L. innocua, and $S$. aureus were incubated for $6,6,11$, and $8 \mathrm{~h}$, respectively. The time-dependent data were collected by the following steps every hour for $5 \mathrm{~h}$. First, utilizing the integrated colony morphology analyzer (ICMA), ${ }^{16}$ colony diameter and elevation were accurately measured for each colony. Second, backwardscatter patterns were captured using the reflection-type instrument. The thickness of the agar for each plate was maintained at $\sim 8 \mathrm{~mm}$.

\subsection{Image Processing and Analysis}

Since the shutter speed is fixed at $2 \mathrm{~s}$, each pattern was captured, cropped to $512 \times 512$ pixels, and converted to gray-scale using the "Luminance" algorithm. ${ }^{19}$ The captured scatter patterns were analyzed by Baclan ${ }^{\mathrm{TM}}$, a stand-alone quantitative imageprocessing software that utilizes different types of features and provides a supervised learning-based classification of the captured pattern. ${ }^{10,20,21}$ After extracting features by rotationally invariant Zernike moments (order 10) and Haralick texture moments (minimum of 1 and maximum of 4 pixels) from each scatter pattern, a classifier was constructed by SVM. Compared to the well-known linear discriminant analysis, SVM is able to construct decision hyperplanes in a multidimensional space that provides the best separation distances among the data classes. ${ }^{22,23}$ For validation, 10-fold cross-validation with repetition of 30 times was performed to calculate the five statistical parameters (PPV, NPV, specificity, sensitivity, and accuracy). Detailed derivation for scatter pattern analysis was previously reported. ${ }^{20,24}$ Using the captured data, an SVM algorithm was 
Table 1 Statistical performance of the Baclan ${ }^{\mathrm{TM}}$ software for the reflection scatter patterns.

\begin{tabular}{lccccc}
\hline & Sensitivity & Specificity & PPV & NPV & Accuracy \\
\hline E. coli & 0.96 & 0.99 & 0.97 & 0.99 & 0.98 \\
L. innocua & 0.92 & 0.99 & 0.96 & 0.97 & 0.97 \\
S. typhimurium & 1.00 & 1.00 & 1.00 & 1.00 & 1.00 \\
S. aureus & 1.00 & 0.98 & 0.94 & 1.00 & 0.99 \\
\hline
\end{tabular}

used for training; the performance of the classifier was provided by the cross-validation matrix. Based on the CV matrix, five statistical parameters were calculated: sensitivity, specificity, positive predictive value (PPV), negative predictive value (NPV), and accuracy (Table 1).

$$
\begin{aligned}
& \text { Sensitivity }=\mathrm{TP} /(\mathrm{TP}+\mathrm{FN}), \\
& \text { Specificity }=\mathrm{TN} /(\mathrm{TN}+\mathrm{FP}),
\end{aligned}
$$

$\mathrm{PPV}=\mathrm{TP} /(\mathrm{TP}+\mathrm{FP})$,

$\mathrm{NPV}=\mathrm{TN} /(\mathrm{TN}+\mathrm{FN})$,

Accuracy $=(\mathrm{TP}+\mathrm{TN}) /(\mathrm{TP}+\mathrm{FP}+\mathrm{TN}+\mathrm{FN})$

where TP, TN, FP, and FN stand for true positive, true negative, false positive, and false negative, respectively.

\section{Experimental Results}

\subsection{Forward- and Backward-Scattering Pattern}

Figure 3 shows a comparison of forward- and backward-scatter pattern, for E. coli $\mathrm{K} 12$ both on a transparent medium (TSA) and opaque mediums (black inked TSA and blood agar plate) The green solid line shows the incoming light, while the red and green dotted lines represent light propagation for the forwardand backward-scatter patterns, respectively. A forward-scattering pattern is observed only with a transparent medium [Fig. 3(c)], whereas the backward-scattering pattern is observed regardless of the opacity of the medium. A red-dashed line shows the (a)

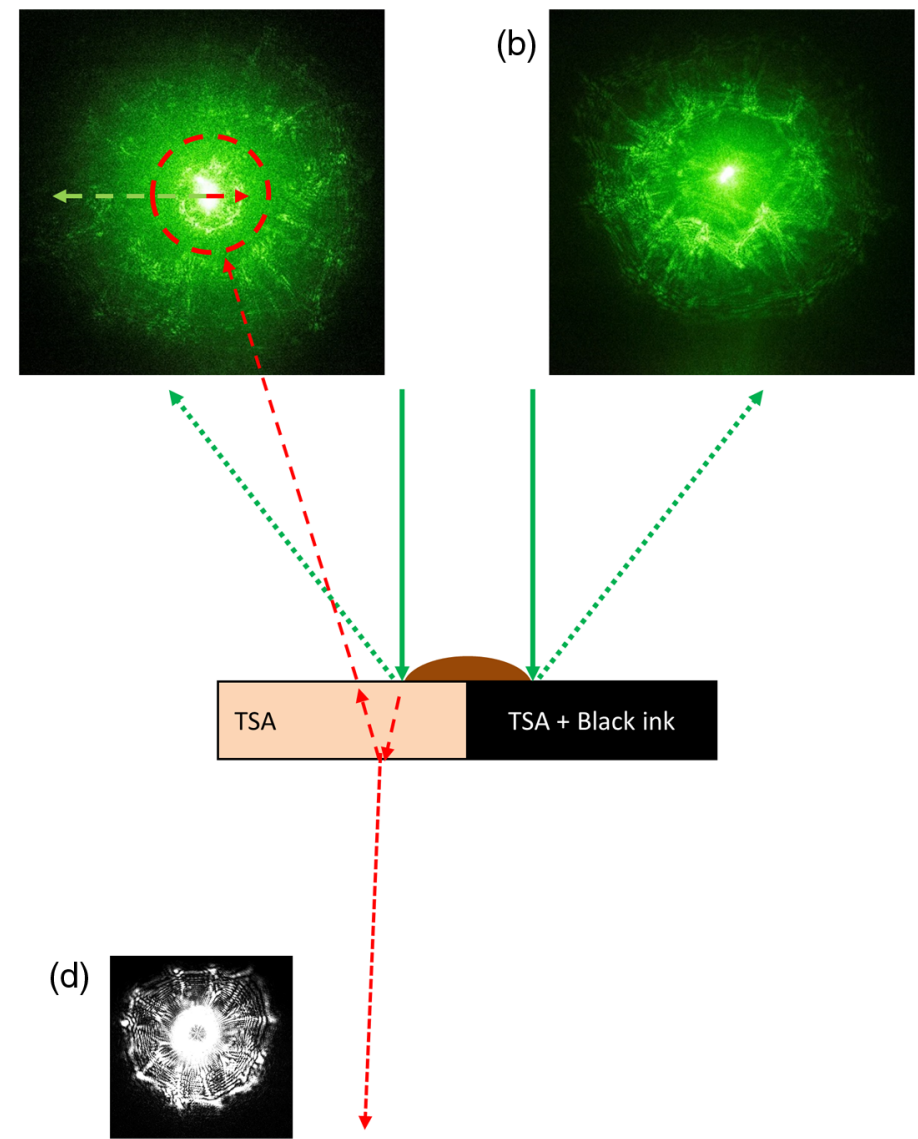

(c)
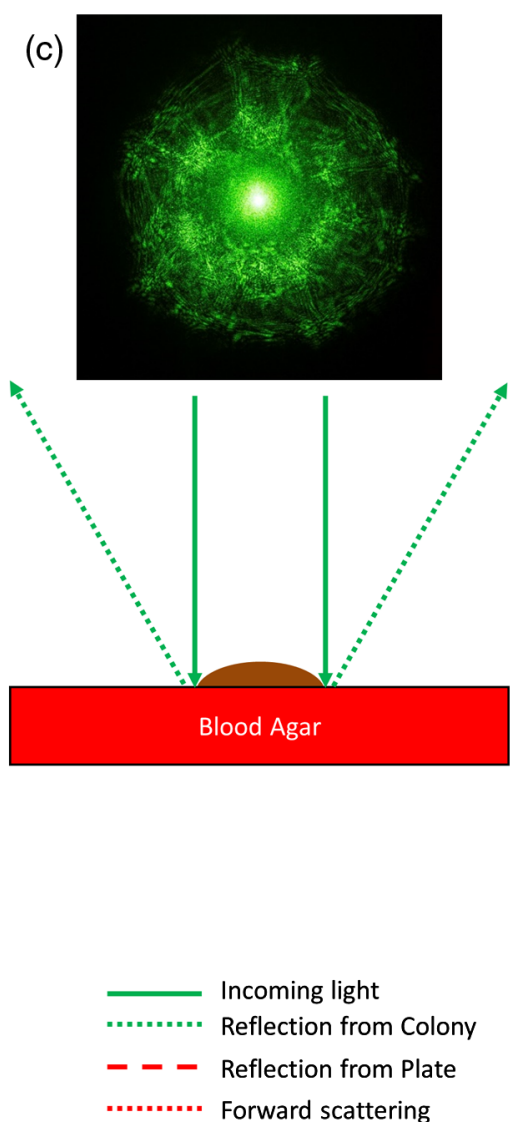

Fig. 3 Comparison of backscatter pattern of bacterial colony (E. coli K12) on both (a) transparent (TSA) and (b) opaque media (black ink added under TSA). For transparent media, both reflection of forwardscatter pattern from the bottom of agar plate (indicated by red-dashed arrow) and actual backscatter pattern (indicated by green-dotted arrow) are captured simultaneously, while only a backward-scatter pattern is captured for opaque media. In both cases, size of a backscattering pattern is larger ( 3.7 times) than the forward-scattering and the reflection of the forward-scattering pattern. (c) The forwardscatter patterns from the same colony on TSA media. In the red-circled area, we can observe the similar pattern of (c) embedded in the backward-scatter pattern. (d) Backscatter pattern from a bacterial colony plated on blood agar media. (blood agar with $5 \%$ sheep blood). 
reflection of the forward-scatter pattern by the Petri-dish surface, which propagates in a backward direction [Fig. 3(a)]. Since the forward-scattering pattern is not generated in the opaque-medium case [Figs. 3(b) and 3(d)], this backward pattern is observed only with transparent media. The shape of the reflection and the forward scattering from the colony are identical. Even when the pattern is observed in the backward direction, however, it is not considered an actual backward-scatter pattern since the origin of the pattern is forward scatter. The half-diffraction angle of the reflection against the incoming laser has a similar value $(0.17 \mathrm{rad})$ to that of its forward-scatter pattern $(0.07 \mathrm{rad})$, which is $\sim 3.72$-fold smaller than the diffraction angle of the backward-scatter pattern (0.633 rad).

\subsection{General Level Backward-Scatter Patterns}

Figure 4 shows snapshots of backward-scatter patterns for the four bacteria genera on 5\% sheep blood agar as measured by the reflective BARDOT system. On visual inspection, the backward-scatter patterns for the genera show distinguishable characteristics. For example, the pattern of E. coli shows an overlapped comb with a spoke-shaped pattern. A small number of ring-shaped patterns appears at the periphery. Unlike other patterns, the boundary between the inner and outer sections of the pattern is unclear. For L. innocua, a speckle cloud with tilde-shaped patterns is observed in the center area, with thin rim patterns with overlapped comb-shaped patterns in the peripheral area. Note that the outermost boundary area of $L$. innocua shows only outward spoke patterns without a circular rim pattern. For Salmonella, very small speckles are observed in

(a)
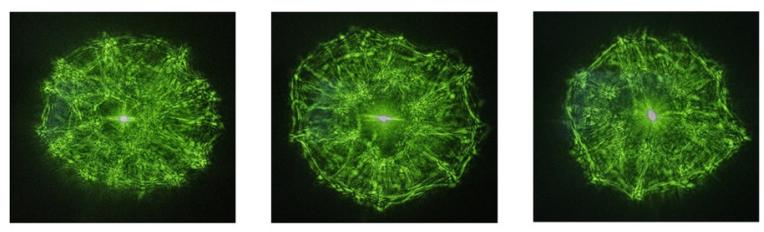

(b)
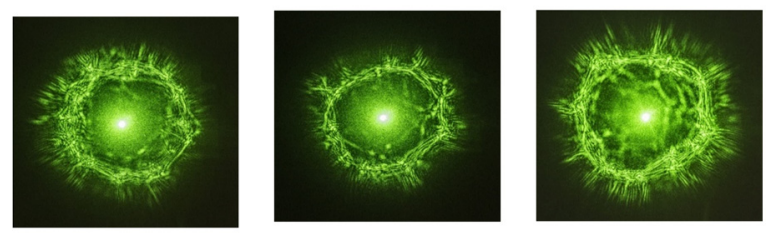

(c)
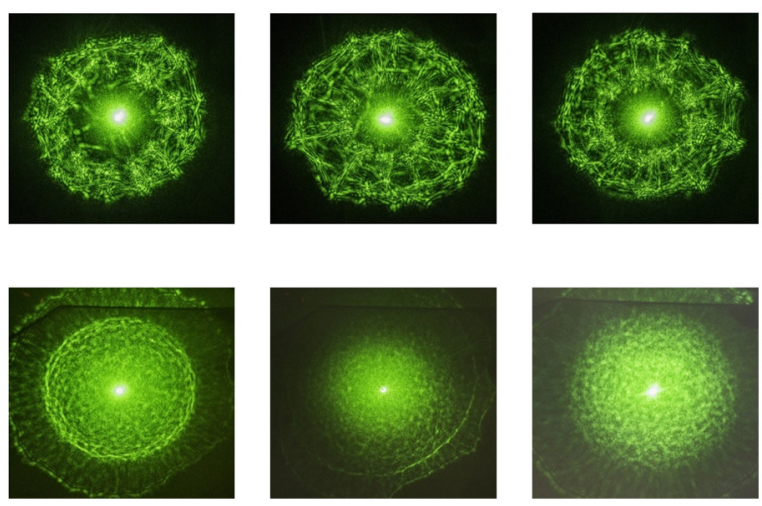

Fig. 4 Representative reflection scatter patterns captured from the reflection-type system. (a) E. coli K12, (b) L. innocua, (c) S. typhimurium, and (d) $S$. aureus. the center area, while relatively thick rims overlapped with spoke patterns are observed at the boundary. For $S$. aureus, interference of the outgoing wave generated the most unique patterns among the four genera tested; center regions were dominated by speckle-like patterns, while the outer rims showed typical ring patterns with irregular edges.

\subsection{Simulation of the Reflected Scatter Dimension}

Figure 5 displays the pattern size predicted from the bacterial colony model. This calculation was based on Eq. $(1)^{5}$ and the maximum normal vector from the surface was used as the pattern size at the imaging plane. Figure 5(a) displays the predicted ray-tracing from the incoming laser assuming that each line represents a photon from the 2-mm-diameter laser source that completely covers the $>1$-mm-diameter bacterial colony. The green-dotted line represents the maximum reflected angle that occurs at the deflection point of the bacterial colony profile. Figure 5(b) shows a close-up of the bacterial colony area. Figure 5(c) displays a comparison of the theoretical calculation and the experimental results for all four organisms tested. Yellow circles represent the geometrical optics model while the three crossbars show the corresponding experimental data for E. coli (red), S. typhimurium (green), and L. innocua (blue). The other crossbar, S. aureus (purple), is a prediction of half diffraction angle based on its aspect ratio, which was not able to be measured due to its excessively larger pattern size. In this study, all four bacterial genera was plated and incubated in order to achieve diameter size of $700 \mu \mathrm{m}$ to $900 \mu \mathrm{m}$. The aspect ratio and diffraction angle are natural characteristics of each genus for a certain growth rate. When the colony diameter size is kept as specified, E. coli, S. typhimurium, and L. innocua result in a smaller aspect ratio of colony along with smaller pattern size. In contrast to the other three, the aspect ratio of $S$. aureus has the irregular characteristic of an enormously large sized pattern due to its large aspect ratio. Obviously, the model indicates the half diffraction angle of reflection is increasing as the aspect ratio of the colony increases. Furthermore, the experiment data also well-correlates to the model in spite of minor error. Therefore, a legitimate pattern size prediction can be made by the model, as large diffraction of $S$. aureus is predicted by its high aspect ratio.

\subsection{Time-Lapse Backward-Scattering Patterns}

Figure 6(a) shows a cross-sectional view of E. coli K12 on blood ager; Fig. 6(b) shows the aspect ratio and pattern size (half diffraction angle). Each profile corresponds to an elevation from a single colony on the same plate with the same incubation time. Variation in diameter and aspect ratio is observed even on the same plate. Once the respective aspect ratio and backwardscatter pattern size are plotted for the colonies tested, we can observe a positive correlation between the aspect ratio and pattern diameter [Fig. 6(b)]. An exception can be noted at E. coli colony \#4. The aspect ratio of the colony is 0.0482 which should have been higher than what is shown. The purple line in Fig. 6(a) is the morphological profile of E. coli colony \#4. The lower aspect ratio can be explained by the tailing edges of the profile at -550 to $-450 \mu \mathrm{m}$ and 400 to $550 \mu \mathrm{m}$ region because the diameter of the colony was overestimated compared to the slope of $E$. coli colony \#4. Therefore, similar to the forward-scatter experiments, ${ }^{25}$ a high aspect ratio produces a larger backward-scatter pattern. Figure 7 displays the time-dependent 
(a)

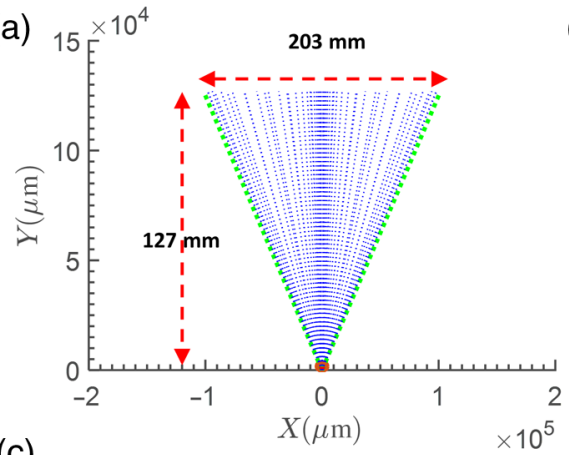

(c)

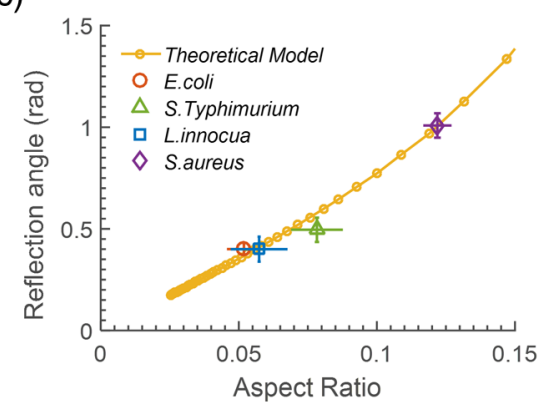

(b)

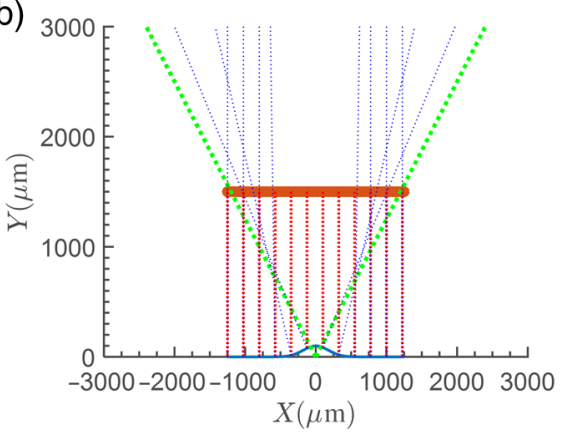

Fig. 5 Theoretical backward pattern size simulation from bacterial colonies by geometrical optic calculation. (a) using Eqs. (1)-(7), laser source is defined as point array of rays across $2 \mathrm{~mm}$ span. Distance from bacterial colony to the screen was set to $127 \mathrm{~mm}$ and patterns size was measured to be $203 \mathrm{~mm}$ in diameter which are provided from the experimental measurements. (b) The close-up view of the reflection phenomena from colony. Orange horizontal line represents the width of the laser and red parallel lines represents the photon arrays in collimated fashion. Blue dashed line shows normal reflection of the incoming photons while green dashed light shows the reflection from the colony surface. (c) Comparison of the aspect ratio and the half diffraction angle. The data clearly indicate a positive correlation between aspect ratio and pattern size. For better comparison, experimental data of aspect ratio and pattern size combination are displayed for four genera.

aspect ratio for all four genera. Starting from time point $t_{0}, 5$ to 15 colonies of each genus at each time point were collected and statistics were calculated. E. coli shows a maximum aspect ratio at $t_{0}+2$ hours and then decreases, while $L$. innocua and $S$. typhimurium plateau after $t_{0}+2$. Meanwhile, $S$. aureus begins with the largest aspect ratio $(0.15)$ and gradually decreases at each time point.

\subsection{Performance of the SVM-Based Classifier}

One interesting result is that the combination of Zernike moments and Haralick texture patterns was critical to ensure high classification rates on the backward-scatter patterns (Fig. 8). In contrast to the forward-scatter patterns, all four genera generate highly disordered patterns in which the Haralick
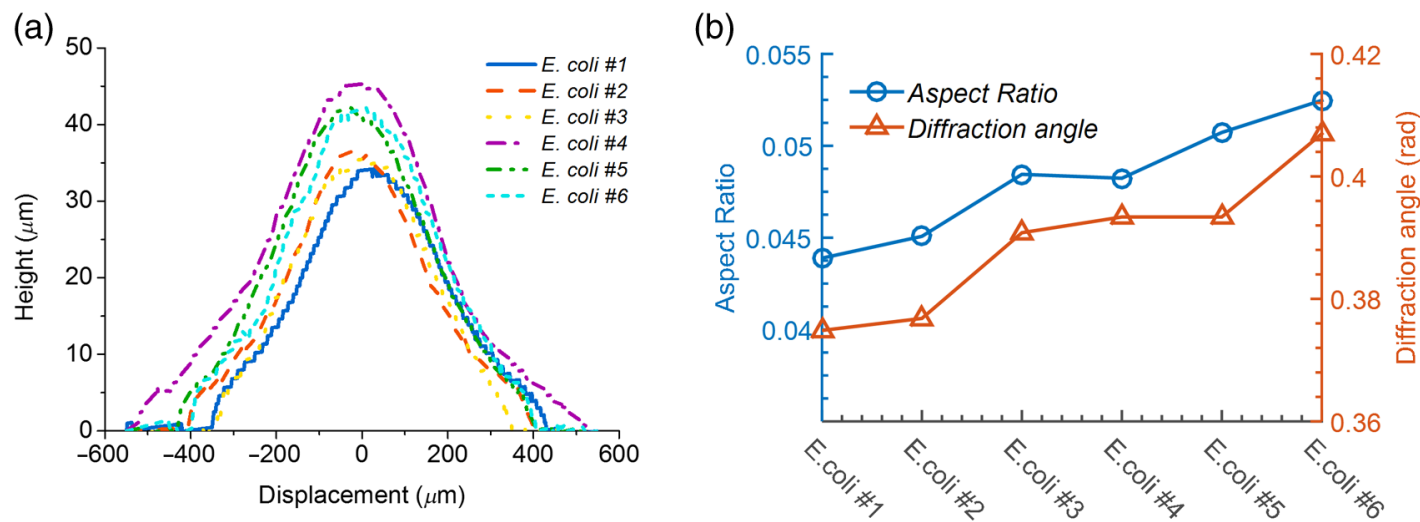

Fig. 6 (a) Quantitative profile measurement data from ICMA using E. coli $\mathrm{K} 12$ at $8 \mathrm{~h}$ incubation as an example. Even in the same plate and same incubation time, their growth morphology can be varying, which results in different diameter and center elevation. (b) The aspect ratio and pattern size [in diffraction angle (rad)] of corresponding colonies of (a). Good correlation is shown between the aspect ratio and pattern sizes. 


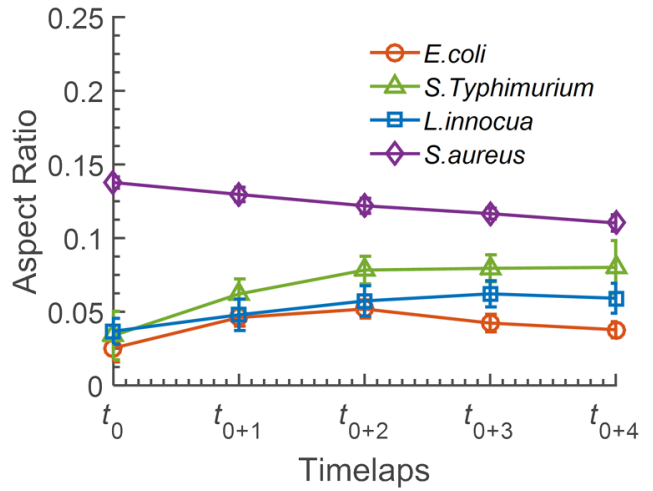

Fig. 7 Statistical aspect ratio variation for all four genera of bacteria for five different time points. After initial measurement, each plate with one genera of bacteria was interrogated with ICMA and reflection scatterometer for every $1 \mathrm{~h}$. Each time point represents the average of 5 to 15 colonies with respective standard deviations. E. coli shows a maximum aspect ratio at $t_{0+2}$ and after that decrease which means colony is expanding in horizontal direction faster than the expansion in vertical direction. S. typhimurium and $L$. innocua both shows initial increase of aspect ratio and plateau after $t_{0+2}$ or $t_{0+3}$ hours. $S$. aureus monotonically decreases its aspect ratio after time $t_{0}$.

texture patterns are contributing to a more accurate classification than the Zernike patterns, which are responsible for circularly symmetric patterns. When the analysis was performed with only the Zernike polynomial, average PPV decreased to $\sim 83 \%$ for Listeria and E. coli. Therefore, all results were obtained using the optimal parameter setting given in Sec. 2.5. Table 1 shows the five statistical parameters calculated from the $\mathrm{CV}$ matrix whose diagonal elements resulted in more than $95 \%$ classification for all four classes. All five statistical measures calculated by Eqs. (6)-(11) provided excellent results of $94 \%$ to $100 \%$ for each genera.

\subsection{Comparison Between Forward-and Backward-Scatter Patterns}

Forward- and backward-scatter patterns from the same microorganisms were compared using two different instruments. Forward-scatter patterns were captured by the standard
BARDOT instrument on TSA media while the backward-scatter patterns were acquired by the reflection-type instrument. Based on the qualitative observation, the former generates more circularly symmetric ring patterns than the latter. For example, forward-scatter patterns of E. coli, L. innocua, and S. typhimurium display dominating ring patterns along with additional radial spoke patterns. In particular, S. aureus generated perfectly concentric ring patterns due to their smooth and high aspect ratio colony profile. Meanwhile, backward-scatter patterns predominantly expressed web-like random interference patterns even though their characteristic length or density was different among the genera. Interestingly, backward-scatter pattern of $S$. aureus was completely different from the rest of the three samples and showed a brighter center and weaker edge portion of the interference patterns.

\section{Discussion}

The backward-scatter patterns of the bacterial colony on blood agar are not effectively generated utilizing a 1-mW, 635-nm laser, which is the standard laser source for the forward scatterometer utilized in the standard BARDOT because the color of the blood agar plate, a major target medium plate, is red owing to the blood in the medium. First, for this wavelength, laser light is highly dispersed and diffusively scattered through the substrate by the blood cells and thus does not generate effective signals in the backward-scatter direction [Fig. 1(c)]. Second, owing to the beam-splitter design, the system is naturally losing half of the incoming power of the laser beam interrogating the bacterial colony. Third, assuming a refractive index of 1.38 to 1.5 for bacterial cells, the Fresnel equation with normal incidence angle provides a reflectance of $2.5 \%$ to $2.8 \%$ only, so the incident power of the laser must be significantly increased to $5 \mathrm{~mW}$ at $532 \mathrm{~nm}$.

Even though only forward-scatter patterns were utilized when interrogating colonies on a transparent medium, by default some signals are reflected backward. However, for transparent media, backward-scatter patterns are composites of both reflection and transmission phenomena. To prove this concept, three media plates were compared against each other: standard TSA, special TSA medium (half transparent + half black ink), and
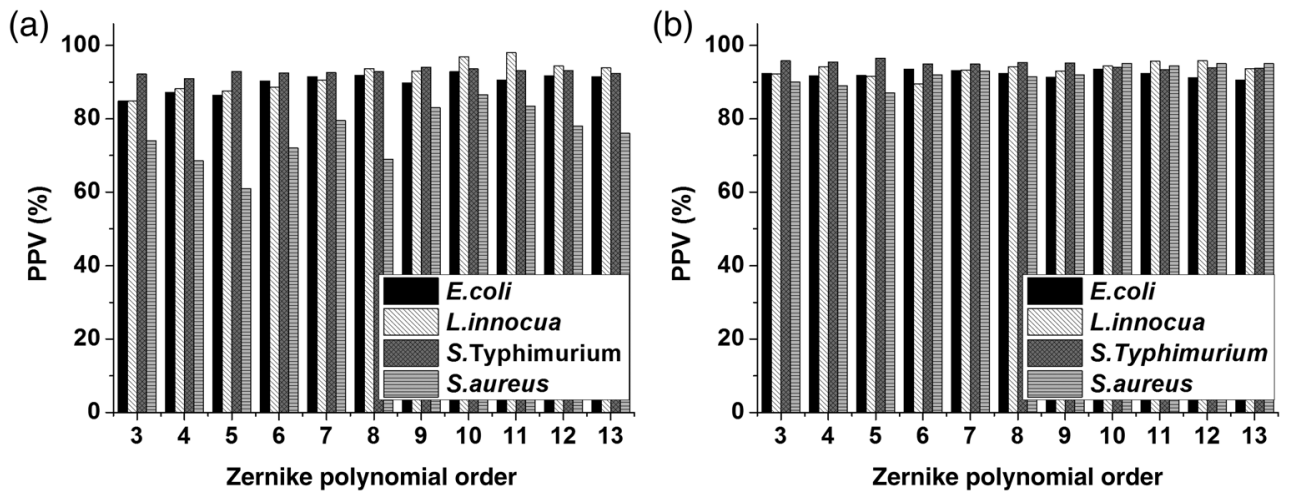

Fig. 8 Comparison of the PPVs (a) without and (b) with the Haralick texture features in order to show the effect of Haralick feature along increase of Zernike polynomial order (3 to 13). Combination of the Zernike polynomial and Haralick texture features clearly increase the correct classification rates for the four genera sample collected by the reflection scatterometer. The Haralick texture feature resulted in a significant difference. However, when Haralick texture feature is applied, the PPV value does not vary much along Zernike polynomial order. Average of $83 \%$ PPVs was achieved without Haralick texture features while the result was boosted to $93 \%$ when the Haralick features were applied. 


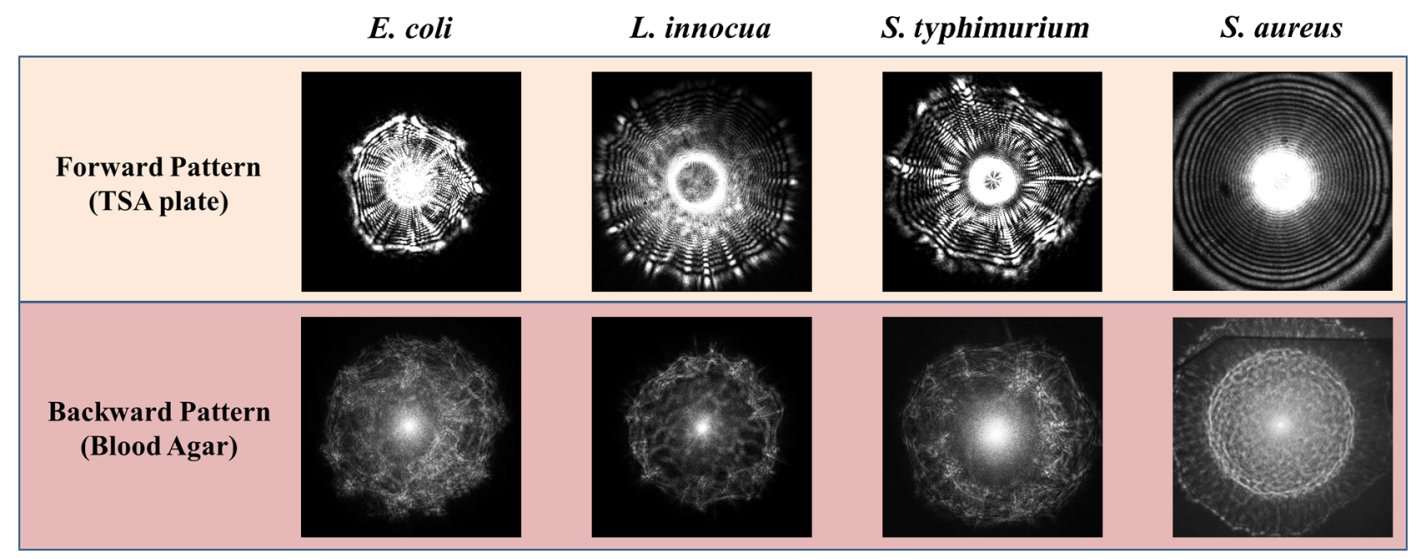

Fig. 9 Comparison of the forward- and backward-scatter patterns. The same samples were incubated on TSA and blood agar and interrogated with BARDOT (transmission) and reflection-type instrument, respectively. Qualitatively, forward-scattering displays the pronounced ring structure while the backscattering patterns are dominated with randomly oriented spokes.

standard blood agar medium. Backward scatter from standard TSA medium generated overlapped scatter patterns with smaller patterns in the center from transmitted scattered light reflected at the bottom of the Petri dish and much larger reflection patterns from the colony surface [Fig. 3(a)]. When we interrogate colonies on the special TSA medium, we can observe that the smaller transmitted scatter patterns have disappeared. This situation is similar to that of the blood agar medium, in which only surface reflected patterns were captured in the backward direction. This situation was quantitatively determined by the laser power meter where optical density (OD) of the blood agar plate was 2.08 ( $0.84 \%$ transmission) and OD of the TSA plate was 0.169 (67.6\% 11 transmission). The higher OD value of blood agar shows that it is difficult to have lights transmitted through the media to generate scatter pattern in forward direction.

The size of forward-scatter pattern increases as it gets farther away from the colony with a half diffraction angle of $\sim 0.1$ rad. ${ }^{11}$ The half diffraction angle of backward-scatter pattern is related to the aspect ratio (ratio of colony center elevation to diameter) as it is in the forward-scatter case. However, the diffraction angle is much larger than the forward diffraction due to the Gaussian profile (convex shape). Owing to the larger diffraction angle of reflected light, both of the imaging sensor size and the distance between the colony and the imaging sensor are the critical design factors. Because of the BS, which is located in between the sample and camera, a minimum distance must be maintained. Therefore, a large screen is utilized instead of an image sensor to capture the full-size pattern with a high-resolution DSLR camera.

The origin of the backward-scatter patterns can be understood as constructive/destructive interference of incoming photons from multiple sources. First, incoming photons are reflected back from the surface of the colony (air-colony interface). Second, the medium-colony interface acts as another source of reflection. Figure 4 shows a stark contrast among the genera in the scatter patterns, which represent the structural and material difference (refractive index) of the colony morphology. For example, $S$. aureus is known to generate a circular and clear-edged colony on blood agar, and the backward-scatter patterns show circularly symmetric ring patterns on the outer rim area of the patterns. There are both macroscopic and microscopic origins on the construction of the backscatter patterns. For example, the curvature and slope of the colony determine the size of the backscatter patterns and center elevation dictates the number of rings observed. ${ }^{11}$ For microscopic reasons, cell arrangement or the cell shape can be a factor that determines the interference patterns. Colony refractive index is known as 1.38 to $1.5^{26-28}$ while agar's RI is not exactly known. However, based on the mixture of agar powder and water, ${ }^{29}$ it will be close to 1.33 to 1.35 range. At this moment, the modeling effect needs a more bottom-up approach to model the microscopic effect into the scatter pattern which will be our future task. Replicating the exact theoretical model in the experiment is somewhat difficult but based on the four genera suggests that the thickness does have some effect on the diffusiveness of the backscatter patterns. However, since the cell shape of $S$. aureus is another factor that can contribute, further development of an individual cell-based optical model will reveal the nature of this phenomena in more detail.

A critical factor related to the differentiability of the backward-scatter patterns is ensuring the capture of whole patterns and not just the center part of the pictures. Even though the image-processing tool (Baclan ${ }^{\mathrm{TM}}$ ) works well with the partial patterns, holistic information of the colony is encoded in the two-dimensional scatter pattern. Compared to the forwardscatter patterns, reflection scatter patterns showed more weblike interference patterns (Fig. 9). Many forward-scatter patterns were dominated by circularly symmetric features where the Zernike polynomial was the crucial analyzer needed, whereas for the backward patterns, the role of Haralick texture features becomes more important to ensure better classification.

\section{Conclusion}

A reflection-type scatterometer is proposed for clinical and veterinary applications of light-scatter technology. The instrument was designed with a BS, a 5-mW, 532-nm laser, and a screen to capture the larger reflection scatter patterns. Four genera of samples were interrogated and provided excellent differentiability based on the combination of Zernike polynomial and Haralick texture pattern analysis.

\section{Acknowledgments}

This material was based upon work supported by the U.S. Department of Agriculture, Agricultural Research Service, under Agreement No. 1935-42000-072. Any opinions, findings, conclusion, or recommendations expressed in this publication 
are those of the author(s) and do not necessarily reflect the view of the U.S. Department of Agriculture. Authors EB and JPR declare interests in Doclu LLC, which develops the next generation data analysis software for ELS system. The terms of this arrangement have been reviewed and approved by Purdue University in accordance with its policy on objectivity in research.

\section{References}

1. P. P. Banada et al., "Optical forward-scattering for detection of Listeria monocytogenes and other Listeria species," Biosens. Bioelectron. 22(8), 1664-1671 (2007).

2. A. K. Singh et al., "Laser optical sensor, a label-free on-plate salmonella enterica colony detection tool," mBio 5(1), (2014).

3. K. Huff et al., "Light-scattering sensor for real-time identification of Vibrio parahaemolyticus, Vibrio vulnificus and Vibrio cholerae colonies on solid agar plate," Microb. Biotechnol. 5(5), 607-620 (2012).

4. K.-P. Kim et al., "Novel PCR assays complement laser biosensor-based method and facilitate listeria species detection from food," Sensors 15, 22672-22691 (2015).

5. Y. He et al., "Rapid identification and classification of Campylobacter spp. using laser optical scattering technology," Food Microbiol. 47, 28-35 (2015).

6. Y. Tang et al., "Light scattering sensor for direct identification of colonies of Escherichia coli serogroups O26, O45, O103, O111, O121, O145 and O157," PloS One 9(8), e105272 (2014).

7. U. Minoni, A. Signoroni, and G. Nassini, "On the application of optical forward-scattering to bacterial identification in an automated clinical analysis perspective," Biosens. Bioelectron. 68, 536-543 (2015).

8. A. Suchwalko et al., "Bacteria species identification by the statistical analysis of bacterial colonies Fresnel patterns," Opt. Express 21(9), 11322-11337 (2013).

9. P. R. Marcoux et al., "Optical forward-scattering for identification of bacteria within microcolonies," Appl. Microbiol. Biotechnol. 98(5), 2243-2254 (2014).

10. B. Rajwa et al., "Discovering the unknown: detection of emerging pathogens using a label-free light-scattering system," Cytometry. Part A 77(12), 1103-1112 (2010).

11. E. Bae et al., "Modeling light propagation through bacterial colonies and its correlation with forward scattering patterns," J. Biomed. Opt. 15(4), 045001 (2010).

12. H. Kim et al., "Scalar diffraction modeling of multispectral forward scatter patterns from bacterial colonies," Opt. Express 23(7), 85458554 (2015).

13. H. Kim et al., "Laser-induced speckle scatter patterns in Bacillus colonies," Front. Microbiol. 5, 537 (2014).

14. L. Beutin, S. Zimmermann, and K. Gleier, "Rapid detection and isolation of shiga-like toxin (verocytotoxin)-producing Escherichia coli by direct testing of individual enterohemolytic colonies from washed sheep blood agar plates in the VTEC-RPLA assay," J. Clin. Microbiol. 34(11), 2812-2814 (1996).

15. D. M. Musher, R. Montoya, and A. Wanahita, "Diagnostic value of microscopic examination of Gram-stained sputum and sputum cultures in patients with bacteremic pneumococcal pneumonia," Clin. Inf. Dis. 39(2), 165-169 (2004).

16. H. Kim et al., "Development of an integrated optical analyzer for characterization of growth dynamics of bacterial colonies," J. Biophotonics 6(11-12), 929-937 (2013).

17. F. L. Pedrotti and P. Leno, Introduction to Optics, 3rd ed., Prentice Hall, Englewood Cliffs, New Jersey (1993).

18. J. A. Shapiro, "Pattern and control in bacterial colony development," Sci. Prog. 76(301-302), 399-424 (1992).

19. C. Kanan and G. W. Cottrell, "Color-to-grayscale: does the method matter in image recognition," PloS One 7, e29740 (2012).

20. E. Bae et al., "Development of a microbial high-throughput screening instrument based on elastic light scatter patterns," Rev. Sci. Instrum. 83(4), 044304 (2012).

21. B. Bayraktar et al., "Feature extraction from light-scatter patterns of Listeria colonies for identification and classification," J. Biomed. Opt. 11, 034006 (2006).
22. C.-C. Chang and C.-J. Lin, "LIBSVM: a library for support vector machines," ACM Trans. Intell. Syst. Technol. 2(3), 1-27 (2011).

23. V. N. Vapnik and V. Vapnik, Statistical Learning Theory, Wiley, New York (1998).

24. B. Bayraktar et al., "Feature extraction from light-scatter patterns of Listeria colonies for identification and classification," J. Biomed. Opt. 11(3), 034006 (2006).

25. E. Bae et al., "Biophysical modeling of forward scattering from bacterial colonies using scalar diffraction theory," Appl. Opt. 46(17), 3639-3648 (2007).

26. A. E. Balaev, K. N. Dvoretski, and V. A. Doubrovski, "Determination of refractive index of rod-shaped bacteria from spectral extinction measurements," in Saratov Fall Meeting 2002: Optical Technologies in Biophysics and Medicine IV, pp. 375-380 (2003).

27. S. J. Hart and T. A. Leski, "Refractive index determination of biological particles," in DTIC Document (2006).

28. P. Liu et al., "Real-time measurement of single bacterium's refractive index using optofluidic immersion refractometry," Proc. Eng. 87, 356-359 (2014).

29. E. Bae et al., "On the sensitivity of forward scattering patterns from bacterial colonies to media composition," J. Biophotonics 4(4), 236243 (2011).

Huisung Kim received his BS degree from Hongik University in Korea and his MS degree from Gwangju Institute of Science and Technology (GIST), in Korea. In 2016, he received his PhD in mechanical engineering at Purdue University. His current research is on bacteria detection using optical light-scattering technique. Before he joined Purdue University, he worked at GIST on development of a fiber type confocal microscope module for integrated microscopes. His research interest involves laser applications, mechanical and optical design and control, signal and image analysis, and instrumentation.

Iyll-Joon Doh is a PhD student in the Mechanical Engineering Department at Purdue University whose research interest involves mechanical and electrical design, precision control, instrumentation, and laser applications in biomedical areas. His current research is on bacteria identification using elastic light-scattering techniques. Prior to beginning the PhD program, he received his master's degree of science in mechanical engineering at Purdue University in 2016.

Jennifer Sturgis received her BS degree from Purdue University and has worked in the Laboratory of Professor J. Paul Robinson for the past 15 years. For 10 years, she managed the imaging facilities of Purdue University Cytometry Laboratories, a university-wide core providing confocal and fluorescence microscopy facilities. She has worked with light-scattering technology for microbial identification for the past 5 years.

Arun K. Bhunia is a professor of food microbiology in the Department of Food Science, Purdue University, and currently conducts research on pathogen detection, employing optical and electrical sensors including protein biochip, light-scattering sensors, fiber-optic sensors, and cell-based sensors. He is also studying mechanisms of pathogenesis for Listeria monocytogenes during the intestinal phase of infection. He is also active in teaching graduate level courses: microbial foodborne pathogens, microbial techniques for food pathogens, and intestinal microbiology and immunology.

J. Paul Robinson is currently the SVM professor of cytomics and professor of biomedical engineering in the Weldon School of Biomedical Engineering, Purdue University. He has published over 160 peerreviewed publications, 30 book chapters, and edited 8 books. His laboratory focuses on diagnostic technology development from single cell analysis through microbial detection systems.

Euiwon Bae is currently a senior research scientist of mechanical engineering at Purdue University. He received his BS degree from Korea University, his MS degree from Korea Advanced Institute of Science and Technology, and his $\mathrm{PhD}$ from the School of Mechanical Engineering at Purdue University, specializing in optical metrology. He has over 30 scientific articles and his research interest includes discrete dipole approximation modeling and developing instruments via light scattering for biological samples. 